\title{
A Radiation Dosimeter Concept for the Lunar Surface Environment
}

\author{
J. H. Adams, M. J. Christl, E. Kuznetsov, T. A. Parnell, G.N. Pendleton, J. W. Watts
}

\begin{abstract}
A novel silicon detector configuration for radiation dose measurements in an environment where solar energetic particles are of most concern is described. The dosimeter would also measure the dose from galactic cosmic rays. In the lunar environment a large range in particle flux and ionization density must be measured and converted to dose equivalent. This could be accomplished with a thick (e.g. $2 \mathrm{~mm}$ ) silicon detector segmented into cubic volume elements "voxels" followed by a second, thin monolithic silicon detector. The electronics needed to implement this detector concept include analog signal processors (ASIC) and a field programmable gate array (FPGA) for data accumulation and conversion to linear energy transfer (LET) spectra and to dose-equivalent (Sievert). Currently available commercial ASIC's and FPGA's are suitable for implementing the analog and digital systems.
\end{abstract}

\section{INTRODUCTION}

The deep space radiation environment poses a challenge for personnel dosimetry due to the wide range of particle fluxes and ionization rates (LET) encountered. The galactic cosmic rays (GCR) have a flux of less than one particle per $\mathrm{cm}^{2}$ per second, with a small heavy ion component contributing about half the dose equivalent. The solar energetic particles (SEP) that pose the principal radiation threat to personnel, especially on surface excursions, have had proton fluxes up to $10^{6} / \mathrm{cm}^{2}$-s, small and variable components of $\mathrm{He}$ and occasionally heavier ions. These hazards have been described in the context of space exploration [1].

The statutory requirements for monitoring crew exposures in LEO have been achieved with passive dosimeter techniques. Active dosimeters will be essential for remote monitoring and future long duration missions. In addition to the present operational instruments such as the Tissue Equivalent Proportional Counters (TEPC) [2] that have been developed and tested in LEO aboard the STS and ISS, several new approaches utilizing silicon detectors are under development [3]-[6].

Manuscript received November 23, 2007. This work was supported by the National Aeronautics and Space Administration.

James H. Adams (email: James.H.Adams@NASA.GOV), Mark J. Christl (email: Mark.Christl@NASA.GOV) and John W. Watts (email:

John.Watts@NASA.GOV) are with NASA/MSFC, Space Science Office

(VP62): NASA/MSFC, Huntsville, AL. 35812,USA

Eugeny N. Kuznetsov (email: Evgeny.N.Kuznetsov@NASA.GOV) and Thomas A. Parnell (Thomas.A.Parnell@NASA.GOV) are with COSPAR at the University of Alabama in Huntsville: University of Alabama in Huntsville, Huntsville, AL. 35899,USA

Geoff N. Pendleton (Geoff.Pendlton@Dynetics.Com) is with Dynetics Inc., Huntsville, AL. 35806. USA

\section{INSTRUMENT CONFIGURATION}

Lunar exploration crews will require portable dosimeters that give accurate dose rates and accumulated dose within and outside of habitats for both SEP and GCR. The heavily ionizing component requires that the linear energy transfer (LET) spectrum be measured or calculated from the measurements [7] so that the biological dose (Sievert) can be determined [8]. To accomplish this we use a novel silicon detector configuration that can operate in these environments and develop a strategy to provide the required information. The configuration builds on experience gained with previously developed pixilated silicon detectors [9]. The detector stack consists of two silicon detectors that are used in coincidence as shown in figure 1.

In the configuration described here the first detector, silicon array (SA), is a $2 \mathrm{~mm}$ thick monolithic silicon detector with a segmented anode. The anode pattern is arranged as $4 \times 4$ elements with each anode measuring $2 \times 2 \mathrm{~mm}^{2}$. This anode pattern taken along with the silicon detector thickness form a contiguous array of sensitive cubic volumes, voxels, with a volume of $8 \mathrm{~mm}^{3}$. Each voxel is an independent sensor with a distinct electronic channel that will measure the energy deposited within its boundaries. The array of voxels can be used independently or in combination through post signal processing to form different detector geometries.

The second element is a monolithic silicon detector that is used to identify particles with sufficient energy to penetrate the SA. The penetration counter (PC) must be capable of reliably detecting minimum ionizing particle (MIP). The thickness of the PC is left as a parameter that will be determined through optimization. For most of our calculations we consider a PC thickness of $0.3 \mathrm{~mm}$. Some possible particle trajectories through the detector are shown in Fig. 1. We consider several operating modes that depend upon whether the primary particle's trajectory is confined to one or several voxels, and whether the particle stops in the SA or registers a signal in the PC. 


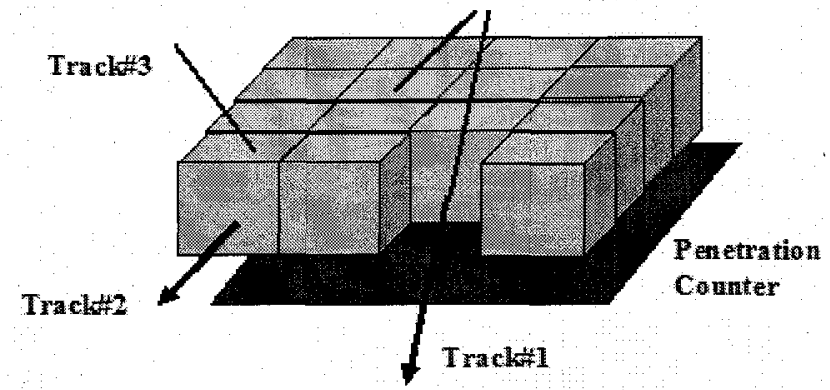

Fig. 1. The dosimeter configuration showing the relative placement of the $\mathrm{SA}$ and $\mathrm{PC}$ and 3 possible trajectories through the detector: Track\#1 intersects a single outer voxel (removed for clarity) and the penetration counter; Track\#2 strikes at least 3 voxels and misses the penetration counter and Track \#3 is stopped in a single voxel.

\section{MEASUREMENT STRATEGy}

The detector response to protons and helium for a wide range of energies has been calculated and is shown in figure 2 . Low energy particles with trajectories confined to and stopping in a single central voxel will have a maximum energy of $30 \mathrm{MeV}$ for protons and $125 \mathrm{MeV}$ for He nuclei. All 4 central voxels in the SA can be used to identify these event types by requiring no coincident signal in the surrounding voxels or PC. For such single-voxel stopping particles the energy of the incident particle can be measured accurately, only limited by small system's noise, but the identification of the particles type is ambiguous over part of the energy range. In both the SEP and GCR environments the composition is mostly protons, $9 \%$ or less of $\mathrm{He}$ nuclei and $1 \%$ or less heavier nuclei. For both low energy stopping particles and relativistic nuclei the relative composition can be found from a histogram of the energy deposited within voxels. Using measured energy spectra and relative composition the LET spectra can be obtained for the measured particle flux using standard look-up tables that will be embedded in the data processing systems. Of course the changing solid angles as a function of energy must be included in the calculated LET spectra. The detection efficiency for an isotropic flux of protons and helium incident on the SA is shown in figure 3.

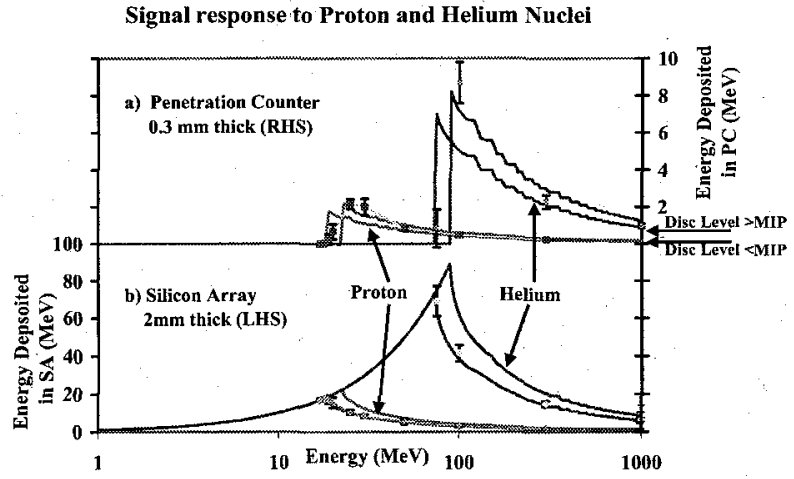

Fig. 2. Energy deposited in the penetration counter (right hand scale) b) Energy deposited in silicon array (left hand scale) for protons and helium nuclei. The energy deposited has been plotted for the minimum and maximum pathlength within a single central voxel. Points with error bars are from
GEANT4 simulations using a computer model of the dosimeter. The simulations used an isotropic flux and selected events with paths only in a single central voxel. The error bars correspond to of the resulting energy deposition distribution. Two discriminator thresholds are used in the PC to separate minimum ionizing particles (MIP).

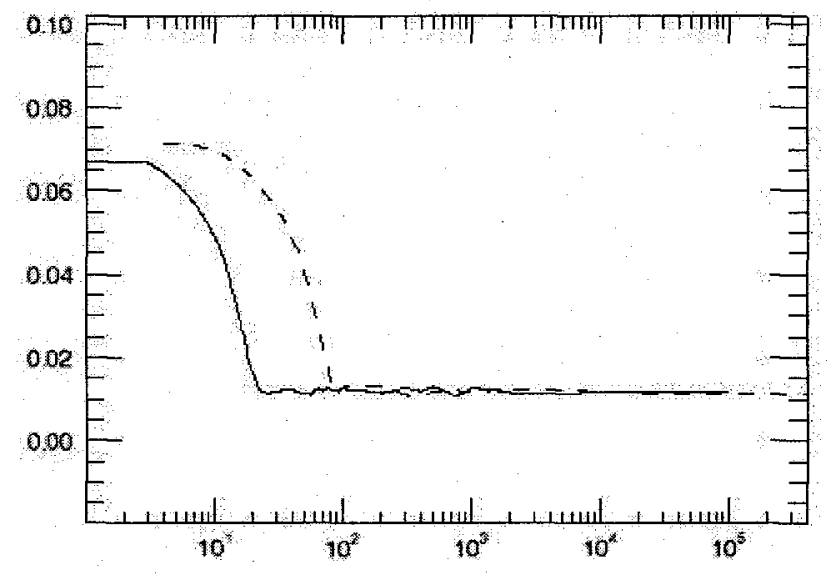

Fig. 3. Calculated single voxel detection efficiency for the dosimeter for proton (solid line) and helium (dashed line) particles as a function of energy. The incident particle flux is isotropic. Both stopping and penetrating particle energies are shown. Axes are relative detection efficiency and primary particle energy (MeV).

For more penetrating particles, including those that pass through more than one voxel, the two detectors comprise a simple charged particle telescope. Figure 2 shows the energy deposition in the SA and PC detectors for single-voxel proton and He nuclei with sufficient energy to reach the PC. For protons with energies between 18 and $30 \mathrm{MeV}$ which penetrate into $\mathrm{PC}$, preliminary calculations have shown that the derived values of LET have an uncertainty up to $\sigma \approx 30 \%$, mostly due to path length differences. At higher energies, between 30 and $100 \mathrm{MeV}$ the uncertainty increases to about $50 \%$, and then improves above $100 \mathrm{MeV}$ where the energy deposition in the SA and PC approximate the LET in silicon. These uncertainties refer to single particle measurements and averages of a sample of particles will be significantly more accurate.

For the GCR nuclei and for the higher energy SEP particles where the energy deposition in the SA and the PC detector closely approximates the LET, particle path length variations in the detectors dominate the accuracy of the measurement. For single cubic voxels the path length variation for isotropic flux compares favorably with other detector shapes as shown in figure 4. The pathlength resolution for a single central voxel with PC signals is $9 \%$. Compared with other detector geometries with resolutions of $35 \%$ (sphere), $59 \%$ (cube) and $51 \%$ (right circular cylinder). For the low GCR flux during quiet periods the selection criteria can be expanded to include events with 2 to 7 voxel hits that have pathlengths resolutions of: $17 \%, 19 \%, 23 \%, 20 \%, 11 \%$ and $7 \%$ respectively. These geometries can be selected based on the trigger scheme planned for this detector. 


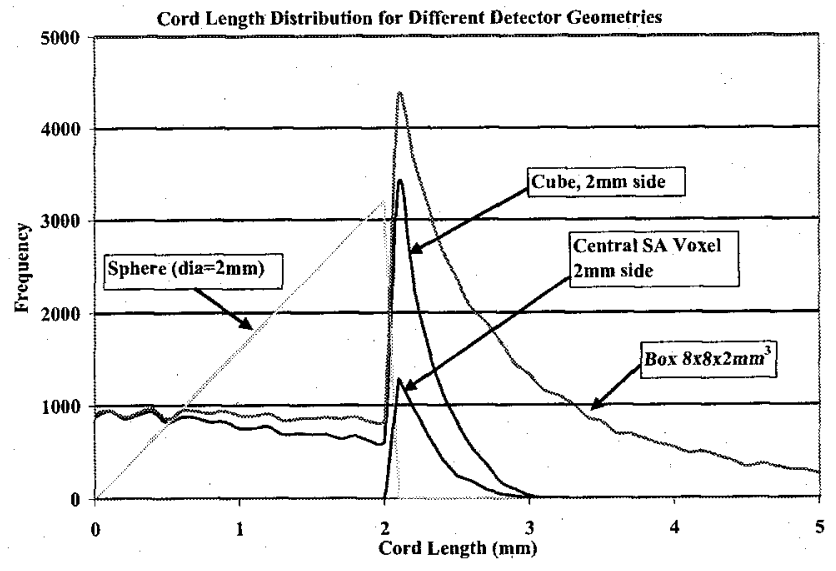

Fig. 4. Isotropic flux cord length distributions for 4 cases: a sphere (dia. $=2 \mathrm{~mm})$, cube $(1=2 \mathrm{~mm})$, Central voxel only $(1=2 \mathrm{~mm})$, and a box $\left(8 \times 8 \times 2 \mathrm{~mm}^{3}\right)$.

The dose measurement for short sampling times will show variations due to counting statistics, path length variations and for intermediate energies the changing energy within the detector volume. For a significant sampling time most of these variations will average out, producing much less error in accumulated dose. Table I \& II show the average event rates per second (SEP) and per day (GCR) for proton and helium primaries respectively. The SEP data uses only the 4 central voxels in the single voxel mode and the GCR data uses the full detector geometry with all possible voxel combinations. These rates are sufficient to reliably establish the dose and dose rates for the two components on the two different time scales.

TABLE I: HELIUM RATES

\begin{tabular}{cccc}
$\begin{array}{c}\text { Energy } \\
(\mathrm{MeV})\end{array}$ & $\begin{array}{c}\text { SEP } \\
\text { (cps) }\end{array}$ & $\begin{array}{c}\text { GCR } \\
\text { Sol. Min } \\
\text { (cpd) }\end{array}$ & $\begin{array}{c}\text { GCR } \\
\text { Sol. Max } \\
(\mathrm{cpd})\end{array}$ \\
\hline $4-20$ & & 166 & 122 \\
$20-100$ & & 48 & 6 \\
$100-500$ & & 72 & 16 \\
$20-500$ & 95 & & \\
$500+$ & & 751 & 475 \\
$500-2000$ & $7 \times 10^{-3}$ & & \\
$2000+$ & $1 \times 10^{-4}$ & &
\end{tabular}

TABLE II: PROTON RATES

\begin{tabular}{cccc}
$\begin{array}{c}\text { Energy } \\
(\mathrm{MeV})\end{array}$ & $\begin{array}{c}\text { SEP } \\
(\mathrm{cps})\end{array}$ & $\begin{array}{c}\text { GCR Sol. } \\
\text { Min } \\
(\text { cpd })\end{array}$ & $\begin{array}{c}\text { GCR } \\
\text { Sol. Max } \\
\text { (cpd) }\end{array}$ \\
\hline $4-40$ & 1406 & 35 & 8 \\
$20-40$ & 49 & 7 & 0.7 \\
$40-300$ & 23 & 286 & 54 \\
$300-1000$ & & 693 & 249 \\
$40-1000$ & 23 & & \\
$300+$ & 0.239 & 1931 & 1082 \\
$1000+$ & $8 \times 10^{-3}$ & 1238 & 833
\end{tabular}

Table I, II Calculated counting rates for helium nuclei (top) and protons (bottom) expected during the SEP in 1989 during the most intense week (counts per second) and the GCR flux at Solar Maximum and Solar Minimum (counts per day) for several energy intervals.

\section{ONGOING WORK}

Studies continue to refine the accuracy of the measurement and strategy for converting from pulse height to tissue dose equivalent. Simulations have been completed at several energies that span the energy range of interest for the common particle species: proton and helium. In particular we are investigating strategies to separate the proton and helium spectra in the regions were the signals in the SA and PC merge (Fig. 5). At low particle energies (larger signals) the overlap region is narrow and the proton and helium signals diverge with increasing energy. At the crossing point (proton 20-22 MeV) the relative contribution for each element can be estimated using data from neighboring points containing only one component. This relative composition is then normalized using data from the merged region.

The respective signals also merge at higher particle energy $\left(E_{\text {proton }}>35 \mathrm{MeV}, \mathrm{E}_{\text {alpha }}>500 \mathrm{MeV}\right)$. At these primary energies the signals are both steadily decreasing with energy. This overlap diminishes as the proton energy approaches $300 \mathrm{MeV}$. At relativistic energies the composition is again readily separated and the average energy loss provides a good estimate of the LET because the detectors are relatively thin. The detector design parameters, especially the thickness of the PC detector, will be optimized to minimize this merged region and to improve the estimated particle flux and composition. 


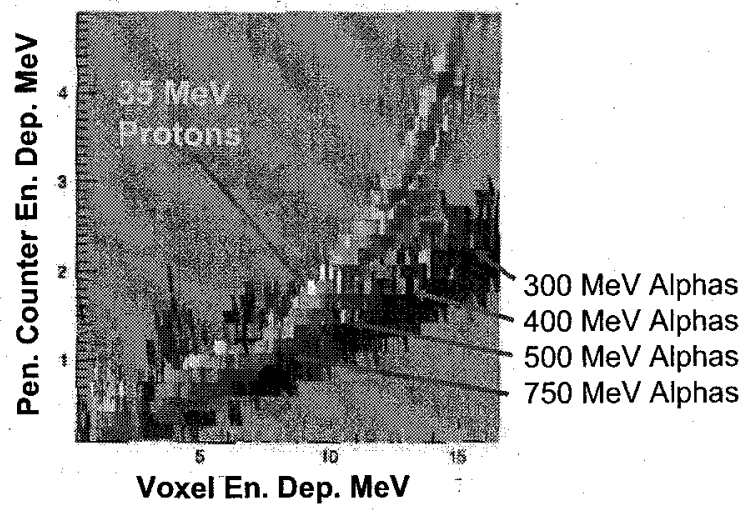

Fig. 5. GEANT4 simulations results for protons (greens, browns) and helium nuclei (blues, reds) at several energy intervals. The lower axis shows the energy deposited in the SA detector while the vertical axis shows energy deposited in the PC detector. Signals from several particle energies are identified by labels. Particle interactions and energy deposited by secondary particles is included

A laboratory bread board of this detector system is being fabricated using high resistivity float zone silicon wafers. The wafer thickness is $2.0 \pm 0.025 \mathrm{~mm}$ and the pixilated anode is $2.0 \times 2.0 \mathrm{~mm}$. The finished detector will be mounted on a transmission style ceramic substrate with wire bonds between the individual voxel elements and gold contacts on the substrate. The detector assembly plugs into sockets mounted on a PCB that contains the front-end-electronics (FEE). The $\mathrm{SA}$ detector is mounted on a printed circuit board (PCB), which has a cut-out beneath the detector to minimize the mass of the intervening material. The PC detector uses fully depleted silicon detectors either 0.3 or $1.0 \mathrm{~mm}$ thick with an active diameter of $10 \mathrm{~mm}$. The $\mathrm{PC}$ is placed in close proximity to the SA to cover the full acceptance geometry.

The two-detector dosimeter as described here has an acceptance solid angle of slightly over one steradian. Although this would satisfy the current specifications for dose measurements for the Constellation program [10], for other applications a larger field of view may be desirable. The dosimeter can be made bi-directional by adding a second $\mathrm{PC}$ on the opposite side of the SA. Configurations that incorporate additional detectors to meet larger fields of view will be examined.

The FEE design will be implemented with the IDEAS VA32 HDR14 ASIC followed by an ADC. The fast output signals from the AISC are used in trigger logic that determines which cells and how many are hit in coincidence. The final trigger is determined from this fast logic input and programmable parameters that permit the acquisition of data during different rates and under different requirements (e.g. GCR versus SEP). The raw signals from the SA and $\mathrm{PC}$ are pulse height analyzed for each trigger. A set of fast discriminators is also contained in the FEE to control the inline processing of the data.
The data acquisition and control will be implemented with a field programmable gate array (FPGA). The proto-type uses a SPARTAN II FPGA with 200,000 gates that is integrated on an PCB interface board with a PC-104 form factor. The FPGA will accumulate data from the $\mathrm{FEE}$, select the processing level based on the data acquisition mode and discriminator outputs, complete the inline processing to convert from the measured quantities to LET spectra, and to tissue dose-equivalent. The FGPA stores the data, performs histograms and calculates the quantities needed for display and telemetry.

\section{REFERENCES}

[1] Space Radiation Hazards, Workshop Report, National Academy Press (2006)

[2] Gersey, B., et al., The response of a spherical tissue-equivalent proportional counter to $56 \mathrm{Fe}$ particles from $200-1000 \mathrm{MeV} /$ nucleon, Rad. Res., 157, 350-360 (2002)

[3] Sakaguchi, T, et al., LET distribution measurements with a new realtime radiation monitoring device-III on board the Space Shuttle STS 84 , NIM A, 437 (1999), 75-87

[4] Narici, L., et al., Study of the radiation environment on MIR space station with SILEYE-2 experiment, Adv. Space Res. Vol. 31, No.1,135(2003)

[5] Roth, D.R.et al., Solid State Microdosimeter for Radiation Monitoring in Spacecraft and Avionics, IEEE Trans. Nuc. Sci., 41 No.6 (1994)

[6] Piscane, V.L., et al., MIDN a spacecraft microdosimeter mission, Radiation Protection Dosimetry, 120, 1-4 (2006), p. 412-426

[7] Adams, D. A., et al., On the use of Lineal Energy Measurements to Estimate Linear Energy Transfer Spectra, submitted to Radiation Measurement (2007),

[8] National Council on Radiation Protection and Measurements, Report No. $142(2002)$

[9] Adams, J.H., et al., The Zero-Degree Detector System for Fragmentation Studies, accepted for Nucl. Instrum. Meth.A., 2006.

[10] NASA Document No. CxP 70024, Section 3.2.7 lonizing Radiation, (2006) 